\title{
Psychiatric aspects of temporal lobe epilepsy before and after anterior temporal lobectomy
}

\author{
Guila Glosser, Alexander S Zwil, David S Glosser, Michael J O’Connor, Michael R Sperling
}

\begin{abstract}
Objectives-Psychopathology has been reported to be prevalent both before and after surgical treatment for medically intractable temporal lobe epilepsy. Individual patients were evaluated prospectively to assess the effect of anterior temporal lobectomy (ATL) on prevalence and severity of psychiatric disease.

Methods-Psychiatric status was assessed in a consecutive series of epilepsy patients before and 6 months after ATL using a structured psychiatric interview, psychiatric rating scales, and self report mood measures.

Results-A DSM-III-R axis I diagnosis was present in $65 \%$ of patients before and after surgery. The most common diagnoses were depression, anxiety, and organic mood/personality disorders. There was a trend for major psychiatric diagnoses to be more common in patients with right compared to left temporal lobe seizure focus, both before and after surgery. The apparent stability in the overall rate of psychiatric dysfunction concealed onset of new psychiatric problems in $31 \%$ of patients in the months shortly after surgery, and resolution of psychiatric diagnoses in $15 \%$ of patients. In the group as a whole, the severity of psychiatric symptoms was lower at 6 months postsurgery than before temporal lobectomy.

Conclusions-The overall prevalence of psychiatric dysfunction was comparably high before and after ATL, but individual changes in psychiatric status and changes in severity of symptoms occurred in many patients in the 6 months after surgery. (F Neurol Neurosurg Psychiatry 2000;68:53-58)
\end{abstract}

Neurology, Jefferson

Medical College,

Philadelphia, PA, USA

D S Glosser

M R Sperling

Department of

Neurosurgery,

Jefferson Medical

College, Philadelphia,

PA, USA

M J O'Connor

Correspondence to:

Dr Guila Glosser,

Department of Neurology

(Gates 3), University of

Pennsylvania Medical

Center, 3400 Spruce Street,

Philadelphia, PA

19104-4283, USA email

glosser@mail.med.upenn.edu

Received 23 February 1999 and in final form

16 July 1999

Accepted 5 August 1999 sectional experimental design versus prospective investigations of the same group of patients examined both before and after anterior temporal lobectomy. To date, most studies of psychiatric outcome after this surgery have been cross sectional and have had little or no information about presurgical psychiatric status. Findings from such investigations must be interpreted cautiously, as it seems likely that psychiatric status before surgery plays an important part in determining postsurgical psychiatric outcome. (2) Another important consideration in the study of psychiatric effects of anterior temporal lobectomy is the time frame during which psychiatric function is assessed. Results may differ, depending on whether the evaluation focuses on lifetime prevalence of psychiatric dysfunction or on psychiatric function within a specific time frame, such as the 6 months immediately preceding surgery. ${ }^{12}$ Psychiatric status in the few months before surgery for epilepsy, when patients are undergoing a complicated evaluation, may not represent their psychiatric function at other periods of their lives. To adequately profile psychiatric status before anterior temporal lobectomy, both current function and functioning over the lifespan must be considered. Similarly, it has been suggested that psychiatric morbidity in the epoch immediately after anterior temporal lobectomy may differ qualitatively and quantitatively from psychiatric problems seen subsequently. ${ }^{13} 14$ (3) Different methods of assessing psychiatric function may also lead to different conclusions. Self reported mood measures ${ }^{15-17}$ are not necessarily comparable with results of psychiatric diagnostic procedures using formal interviews of patients and families or rating scales completed by a psychiatrist. ${ }^{12}{ }^{13}$ Psychiatric diagnosis must consider not only the transient mood states that are most often captured in self report mood questionnaires, but the full context of the presenting symptoms including the history, other medical-behavioural characteristics, and findings by people other than the patient. In patients with neurological disorders self report measures of mood must be interpreted with special caution, as some neurological disorders differentially affect how patients perceive, interpret, and report their affective states. $^{18} 19$

Obviously, it is difficult to consider all of the aforementioned issues in a single investigation. The study reported below, however, was designed to control for at least some of these factors. Psychiatric functioning was assessed prospectively, before and after surgery, in a group of patients undergoing anterior temporal lobectomy. Formal psychiatric diagnoses were 
Table 1 Demographic and medical characteristics of the 39 patients with temporal lobectomy who completed presurgical and postsurgical psychiatric evaluations and five patients who did not return for postsurgical psychiatric follow up

\begin{tabular}{llll}
\hline & LTL $(n=16)$ & RTL $(n=23)$ & Dropout (n=5) \\
\hline Age at surgery (mean (SD)) & $31.5(6.7)$ & $32.9(8.7)$ & $32.4(10.0)$ \\
Age at first risk (mean (SD)) & $5.8(10.0)$ & $8.6(9.1)$ & $14.6(17.3)$ \\
Age at seizure onset (mean (SD)) & $11.5(10.3)$ & $14.8(9.6)$ & $15.8(16.3)$ \\
Percent male & $63 \%$ & $48 \%$ & $40 \%$ \\
Years of education (mean (SD)) & $12.4(1.6)$ & $13.3(2.5)$ & $13.0(1.7)$ \\
WAIS-R full scale IQ (mean (SD)) & $88.7(13.8)$ & $92.2(15.0)$ & $88.8(7.5)$ \\
\hline
\end{tabular}

established using standardised criteria based on a semistructured psychiatric interview as well as information from family members and other medical personnel. In an attempt to capture a broad time frame, lifetime diagnoses as well as current psychiatric diagnoses were ascertained. Together with the determination of psychiatric diagnoses, quantitative psychiatric ratings were obtained for each patient, and patients themselves completed standardised self report measures of mood.

\section{Methods}

SUBJECTS

All patients admitted to the monitoring unit of a comprehensive epilepsy centre for evaluation of surgical treatment of medically intractable TLE over an 18 month period were entered into the study. After neurodiagnostic evaluation, consisting of non-invasive EEG, MRI, neuropsychological testing, intracarotid amobarbital test, and intracranial EEG monitoring in selected cases, ${ }^{20} 2144$ patients underwent anterior temporal lobectomy. Table 1 presents demographic and medical characteristics of the 39 patients who returned for psychiatric re-evaluation after surgery, and the five patients who refused psychiatric follow up after surgery. Analyses of variance and $\chi^{2}$ statistics indicated that those who completed the study did not differ from those who dropped out on any background variables nor in presurgical rates of psychiatric diagnoses. Among study completers there were no significant differences on any background variables between those who underwent left (LTL) and right (RTL) temporal lobectomy.

\section{PROCEDURES}

Patients were examined psychiatrically during admission for scalp/sphenoidal video/EEG monitoring, a mean of 3.9 months before surgery. Axis I diagnoses, based on the criteria of the Diagnostic and statistical manual of mental disorders, third edition, revised (DSM-III-R), ${ }^{22}$ were determined after an interview modelled on the structured clinical interview for DSMIII-R (SCID), ${ }^{23}{ }^{24}$ review of all available psychiatric and neurological records by a board certified psychiatrist, and in many cases interview of a family member. In determining DSM-III-R diagnoses in this patient population, it is often difficult to establish whether an "organic" factor may be modifying the psychiatric presentation. Rather than assuming that all psychopathology relates to the epilepsy, a diagnosis of an organic psychiatric disorder was made only when there was a clear temporal association between the onset of psychiatric symptoms and factors such as medication change, recent seizure flurry, or coincident surgery. For each patient, current diagnoses for the 6 months preceding the evaluation were determined, along with diagnoses present at any time during life before surgery. The psychiatrist also completed the global assessment of function scale (GAF) on axis V of the DSM-III-R, and the brief psychiatric rating scale (BPRS).$^{25} \mathrm{At}$ about the same time as the psychiatric interview each patient also completed the following self report scales: state-trait anxiety inventory $(\mathrm{STAI})^{26}$; Beck depression inventory $(\mathrm{BDI})^{27}$; profile of mood states (POMS) ${ }^{28}$ In all cases the interviewing psychiatrist was blinded to the results of the psychiatric rating scales.

Each patient was re-evaluated psychiatrically a mean of 7.1 months after anterior temporal lobectomy using the same interview, rating, and self report procedures conducted before surgery. As it is the practice in this epilepsy centre not to change the anticonvulsant regimen in the first year after surgery for epilepsy, virtually all patients were on the same medications when evaluated before and after surgery.

Because of scheduling difficulties and reading problems, 10 patients had incomplete data on one or more of the self report scales.

\section{Results}

Consistent with previous studies, we found that, as a group, patients undergoing anterior temporal lobectomy were highly symptomatic psychiatrically both before and after surgery. Combined data from presurgery and postsurgery psychiatric evaluations indicated that $77 \%$ of the patients met criteria for an axis I DSMIII-R diagnosis sometime in their life either before or after surgery (table 2). There was no difference in the overall proportion of patients who met criteria for an axis I DSM-III-R diagnosis for the 6 to 12 months before the presurgical psychiatric evaluation and for the 6 to 12 months after anterior temporal lobectomy $\left(\chi^{2}\right.$ $=0.05 ; \mathrm{p}>0.10$ ).

Mood disorders (major depression, dysthymia, anxiety disorders, and organic mood disorders) accounted for most of the psychopathology in this patient group (table 3). A smaller, but still remarkable, proportion of patients presented with organic personality disorders and substance misuse disorders, especially before surgery. Many of the patients presented with complex mood and personality disorders that included mixed features of depression, anxiety, irritability, and mood lability similar to the pleomorphic affective disorder described by Blumer et al. ${ }^{1}$ Stable interictal psychotic disorders were notably

Table 2 Proportions of patients with temporal lobectomy with any DSM-III-R axis I lifetime psychiatric diagnosis, either before or after surgery, and those with an active diagnosis in the 6 or so months before surgery and in the 6 months or so after temporal lobe surgery

\begin{tabular}{llll}
\hline & RTL & LTL & All patients \\
\hline Lifetime: pre or postsurgery & 0.83 & 0.69 & 0.77 \\
Six months presurgery & 0.65 & 0.31 & 0.51 \\
Six months postsurgery & 0.65 & 0.44 & 0.56 \\
\hline
\end{tabular}


Table 3 Proportions of patients with temporal lobectomy with specific DSM-III-R axis I psychiatric diagnoses any time prior to surgery, in the 6 or so months before surgery and in the 6 or so months after temporal lobe surgery

\begin{tabular}{|c|c|c|c|c|c|c|}
\hline & \multicolumn{3}{|c|}{ Right temporal lobectomy } & \multicolumn{3}{|c|}{ Left temporal lobectomy } \\
\hline & $\begin{array}{l}\text { Any time } \\
\text { presurgery }\end{array}$ & $\begin{array}{l}6 \text { Months } \\
\text { presurgery }\end{array}$ & $\begin{array}{l}6 \text { Months } \\
\text { postsurgery }\end{array}$ & $\begin{array}{l}\text { Any time } \\
\text { presurgery }\end{array}$ & $\begin{array}{l}6 \text { Months } \\
\text { presurgery }\end{array}$ & $\begin{array}{l}6 \text { Months } \\
\text { Postsurgery }\end{array}$ \\
\hline Major depression/dysthymia & 0.31 & 0.22 & 0.13 & 0.13 & 0.13 & 0 \\
\hline Bipolar/cyclothymia & 0.09 & 0.09 & 0.04 & 0 & 0 & 0 \\
\hline Organic mood & 0.26 & 0.09 & 0.35 & 0.38 & 0.06 & 0.25 \\
\hline Anxiety disorders & 0.22 & 0.09 & 0.13 & 0.13 & 0.06 & 0.06 \\
\hline Organic personality & 0.22 & 0.17 & 0.09 & 0.13 & 0.13 & 0 \\
\hline Substance/alcohol abuse & 0.17 & 0 & 0.04 & 0.06 & 0 & 0 \\
\hline Psychotic & 0.04 & 0 & 0.04 & 0.06 & 0.06 & 0.06 \\
\hline Other & 0.13 & 0.22 & 0.22 & 0.06 & 0 & 0.06 \\
\hline
\end{tabular}

absent in this sample of patients, because such patients were mostly screened out during presurgical assessment on the assumption that they are less likely to improve functionally after anterior temporal lobectomy. ${ }^{29}$

Tables 2 and 3 show that at all time periods patients with RTL were more symptomatic psychiatrically than patients with LTL. The difference in proportions of patients with RTL or LTL with major psychiatric diagnoses was marginally significant for the 6 months before the presurgical psychiatric evaluation $\left(\chi^{2}=3.10\right.$; $\mathrm{p}=0.07)$. Although patients with RTL also showed a trend for higher rates of lifetime and postsurgical psychiatric dysfunction, these differences were not statistically significant ( $p$ values $>0.10$ ). The effects of language dominance on psychiatric functioning within the RTL and LTL groups could not be examined reliably because of the relatively few non-left hemisphere language dominant patients.

Despite apparent stability of psychiatric dysfunction in the anterior temporal lobectomy group as a whole, individual patients showed changes in psychiatric status after surgery. Twelve $(31 \%)$ of the patients (eight RTL and four LTL) experienced onset of a new psychiatric diagnosis or exacerbation of a pre-existing diagnosis that had been determined to have been in remission before surgery. Ten of these patients developed a rapidly cycling mood disorder with mixed features of depression, anxiety, and irritability, which tended to occur in relatively brief episodes or cycles lasting hours or days. ${ }^{30}$ The affective disturbance in these patients was rated as having an "organic" basis as it was related in time to the epilepsy surgery. Symptomatic patients reported that the mood disturbance began 1 to 4 weeks after surgery. Most were still reporting some symptoms when seen for the 6-7 month postsurgical psychiatric evaluation, but symptoms resolved completely for all but two patients by the time they returned for neuropsychological reevaluation about 1 year after surgery. By contrast with patients who developed new symptoms after surgery, there were another six $(15 \%)$ who improved psychiatrically (four RTL and two LTL). All of these patients had exhibited mood dysfunction before surgery, but in the 6-12 months after surgery they no longer exhibited symptoms of a DSM-III-R axis I diagnosis. Finally, of 21 patients whose psychiatric status did not change after surgery, eight continued to be symptomatic with the same diagnoses as were present immediately before surgery, and the remaining patients were asymptomatic both before and after surgery.

Of the five patients who did not return for formal psychiatric evaluation after surgery, three remained stable psychiatrically and two are thought to have experienced transient postsurgical mood dysfunction, based on their reports to physicians during medical follow up 1-2 years after surgery.

Because of the importance of psychiatric symptoms in managing patients clinically after anterior temporal lobectomy, we examined various factors that might be associated with changes in psychiatric status in the immediate postsurgical period: patients whose psychiatric state worsened after surgery did not differ from the other surgical patients in terms of background characteristics (age at surgery, at seizure onset and at first risk, and intelligence) nor presurgical psychiatric ratings on the BPRS or mood self ratings (POMS, Beck, STAI). The proportions of patients with changed psychiatric status after surgery were not different between RTL and LTL groups $\left(\chi^{2}=0.81\right.$; $\mathrm{p}>0.10)$. There was no significant relation between change in psychiatric status after surgery and seizure status at 6 months after surgery. The postsurgical psychiatric state of patients who were completely seizure free (class I according to Engel's ${ }^{31}$ criteria) did differ from those who were not seizure free 6 months after surgery $\left(\chi^{2}=1.55 ; p>0.10\right.$, table 4 .

Comparison of GAF and BPRS ratings before and after surgery (table 5) indicated a significant postoperative increase in the highest level of adaptive functioning (GAF) ( $t$ $(38)=2.55 ; \mathrm{p}<0.05)$ and an overall reduction in psychiatric symptoms on the BPRS $(t$ $(38)=1.85 ; p=0.07)$. Thus although the prevalence of psychiatric disease did not change at 6 months after anterior temporal lobectomy, across all patients there was a significant decline in the severity of symptoms rated by the psychiatrist compared with presurgical ratings.

Table 4 Change in psychiatric status postsurgery and seizure free status at 6 months post-temporal lobectomy

\begin{tabular}{lcl}
\hline & $\begin{array}{l}\text { Seizure free } \\
(n=31)\end{array}$ & $\begin{array}{l}\text { Not seizure } \\
\text { free }(n=8)\end{array}$ \\
\hline $\begin{array}{l}\text { Psychiatrically improved } \\
\text { Psychiatrically unchanged }\end{array}$ & 4 & 2 \\
Psychiatrically worse† & 17 & 5 \\
\hline
\end{tabular}

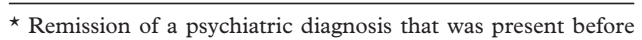
surgery.

† Onset of a new psychiatric diagnosis or exacerbation of a preexisting psychiatric diagnosis that had been in remission before surgery. 
Table 5 Global assessment of function ( $G A F)$ rating of highest function in the past 6 months and brief psychiatric rating scale (BPRS) ratings before and after temporal lobe surgery

\begin{tabular}{lll}
\hline & Presurgery & Postsurgery \\
\hline GAF (DSM-III-R) ${ }^{\star}$ & $73.2(15.7)$ & $77.4(14.2)$ \\
BPRS† & $27.1(7.2)$ & $24.9(7.2)$ \\
\hline
\end{tabular}

$\star$ A higher score indicates less psychiatric symptomatology. tA lower score indicates less psychiatric symptomatology.

Table 6 Self report ratings of mood before and after temporal lobe surgery lower scores indicate less mood dysfunction)

\begin{tabular}{lll}
\hline & Presurgery & Postsurgery \\
\hline Depression (BDI) & $10.3(9.2)$ & $6.3(8.4)$ \\
Anxiety (STAI-state) & $38.8(12.2)$ & $35.0(11.2)$ \\
Anxiety (STAI-trait) & $42.4(10.3)$ & $34.8(10.9)$ \\
Total mood disturbance (POMS) & $25.6(37.6)$ & $18.0(36.8)$ \\
\hline
\end{tabular}

Finally, consistent with severity ratings by the psychiatrist, comparisons of patient self report ratings of mood before and after surgery (table 6) indicated significant reductions in anxiety (STAI-trait), depression (BDI), and overall mood dysfunction (POMS total mood disturbance) postsurgery ( $\mathrm{p}$ values $<0.05$ ). The RTL and LTL groups did not differ significantly on any self report measures, either before or after surgery.

\section{Discussion}

The prevalence of major psychiatric disorders found in this sample of patients is high and comparable with that reported in previous studies of patients with intractable TLE seen in surgical epilepsy centres ${ }^{1-3} 7814$ (see KochWeser $e t a l^{4}$ and Naylor et $a l^{2}$ ). About one half of all patients had an active major psychiatric diagnosis in the 6 months preceding the assessments before and after anterior temporal lobectomy, and three quarters of all patients had met criteria for an axis I DSM-III-R diagnosis sometime in life.

Our results concur with the findings of Blumer and others ${ }^{1812}$ that both before and after surgery mixed mood disorders are the most common interictal psychiatric problems among patients with TLE. Although we attempted to apply conventional psychiatric diagnoses to our patients, we found that many patients did not fit easily into a single standard (DSM-III-R) diagnostic category. Rather, most patients were best characterised by a symptom complex involving mixed features of depression, anxiety, and irritability that tended to occur often, but intermittently. Episodic cycles of affective disturbance tended to be brief, lasting less than 48 hours. This presentation is most similar to the pleomorphic, intermittent, rapid cycling "interictal dysphoric disorder", which has been defined by the presence of at least three of the following symptoms: depressed mood, anergia, irritability, pain, insomnia, fears, anxiety, and euphoric moods.

Occurrence of new mood disorder in the immediate postoperative months has been reported by several investigators, ${ }^{12}{ }^{33} 34$ leading one research group to suggest that " ... it would be reasonable to warn all patients and their families before surgery that they may experi- ence a brief increase in emotionality in the weeks after the operation". ${ }^{14}$ We found that in the first few weeks after surgery, about one quarter of patients experienced either new psychiatric symptoms or clear exacerbation of preexisting psychiatric symptoms. Symptoms were expressed mostly as a rapid cycling mixed mood disorder. In general, the mood disorder was transitory, and it cleared in almost all patients by the time that they were seen for neuropsychological re-evaluation 1 year after surgery. The reasons for the occurrence of this transient postsurgical mood disorder remain unclear. Some investigators have speculated that psychiatric problems after anterior temporal lobectomy may be a consequence of disrupted functioning within temporal lobe structures, such as the amygdala, which are known to be involved in the modulation and expression of emotion. ${ }^{14}$ Others have considered that "forced normalisation" leads to affective disturbance, through suppression of the antidepressant effects of seizures. ${ }^{12}$ We found a weak, non-significant, trend in our data supporting the idea that seizure free patients may be at higher risk for developing transient psychiatric worsening in the short term after temporal lobectomy, but we do not have long term seizure outcome. It has also been suggested that psychiatric problems after surgery occur because patients may be responding negatively to increased expectations for new role functions once seizures have been abolished or reduced. ${ }^{14}$ Alternately, it may be that patients with TLE, many of whom have had lifelong adjustment and psychiatric problems and limited coping skills, become more symptomatic in response to the psychological trauma and physiological stress that is normally associated with neurosurgery. Clearly, the factors underlying changes in psychiatric status shortly after anterior temporal lobectomy require further investigation, if we are to advance our capacity to anticipate and treat this common problem.

The variables relating to psychiatric symptoms occurring early after surgery are probably different from those associated with later psychosocial adjustment. Although the relation between seizure control and psychiatric symptoms shortly after surgery is still an open question, seizure free status is recognised to be the most powerful predictor of improved psychiatric and psychosocial adjustment outcome later in the postoperative course. ${ }^{7}{ }^{15335}$ Persistence of seizures, for example, is highly predictive of the development of newly occurring psychiatric disorders in patients who are more than 5 months after surgery. ${ }^{12}$ Similarly, despite an absent relation between psychiatric status preoperatively and psychiatric difficulties in the early postoperative period, it has been shown convincingly that preoperative psychosocial adjustment is an important predictor of long term postoperative psychosocial outcome even in patients with good seizure outcome. ${ }^{15} 34$

Prior studies suggest that psychotic disorders are the least likely to change after anterior temporal lobectomy. ${ }^{37936}$ This finding has led to the general practice of not offering surgical 
treatment to patients with epilepsy and psychotic disorders, especially when the psychiatric problem is a major determinant of the patient's disability. On the other hand, there have been several reports suggesting that, in the long term, psychiatric symptoms consisting of irritability and aggressive behaviours do improve after anterior temporal lobectomy. ${ }^{79} \mathrm{We}$ did not examine the second types of symptoms specifically, but it is possible that the reduced severity of mood dysfunction found 6-7 months after surgery reflects fewer symptoms of anger.

Although most patients we studied remained symptomatic psychiatrically in the months after anterior temporal lobectomy, psychiatric symptoms did remit in a small proportion of patients after surgery. Even in patients who remained symptomatic after surgery, overall severity of psychiatric symptoms, level of adaptive functioning, and self rated mood all improved significantly compared with the presurgical assessment. This psychiatric improvement may represent relief from the stressful anticipation of surgery, the naturally cycling course of the interictal affective disorder in TLE, or a more permanent change in neuropsychiatric status resulting from surgery itself. More detailed studies extending over longer time periods are still required to chart the course of affective disorders in patients after anterior temporal lobectomy.

With regard to laterality of the neurological dysfunction in patients with TLE, it is generally accepted that schizophrenic-like psychoses are more common before surgery in patients with left or language dominant temporal lobe seizure focus. ${ }^{72}{ }^{37}$ Interestingly, after surgery it seems that new onset psychotic disorders are actually more common in patients with right temporal lobe seizure focus. ${ }^{36}$

There has been less agreement about the relation of laterality of seizure focus to mood disorders. Some studies have found no relation, ${ }^{1}$ some have reported higher rates of depression among patients with left TLE, ${ }^{13} 18$ whereas other studies, ${ }^{2} 78$ including the present one, have found that serious affective dysfunction occurs more often in patients with right temporal lobe seizure focus, when rated using standardised psychiatric diagnostic criteria. At all time periods we found patients with RTL to be more symptomatic in terms of affective disorders than patients with LTL, although the relative rate of change in psychiatric status after surgery was comparable in both groups. Despite apparent differences between right and left TLE in the psychiatrist's rating of symptoms, there were no differences in self reported mood between groups, suggesting that even when it seems to observers that patients with right TLE have more mood dysfunction, these patients themselves do not necessarily make the same attribution. ${ }^{19}$ Patients with right hemispheric dysfunction are known to be relatively less accurate in their perception and interpretation of emotional cues in general ${ }^{39}$ and this may extend to misattributions of their own emotional states.
When interpreting the results of this study, several caveats must be kept in mind. Firstly, our study sample consisted of a highly selected group of patients. Whereas there are certain advantages to studying surgical patients with precise documentation of neurological dysfunction, we also recognise that this subgroup does not represent the full range of patients with TLE. The stringent selection criteria for undergoing anterior temporal lobectomy limits the total number of patients available for study. A larger group of patients is required to adequately evaluate the complex associations among various neurobiological and psychosocial variables determining psychiatric function in patients with TLE. Another limitation of this study, as well as prior studies of psychiatric function in patients with TLE, derives from the fact that many standard psychiatric evaluation measures are not necessarily appropriate for patients with epilepsy. It is becoming increasingly clear that some of the most common and disabling psychiatric problems in patients with TLE cannot be classified easily using conventional psychiatric diagnoses. ${ }^{18}$ Fortunately, difficulties using DSM-III-R criteria for diagnosing affective disorders in patients with TLE have been partly remedied by the revised DSM-IV, ${ }^{40}$ which specifies that a "mood disorder due to a general medical condition" (formerly termed "organic") may include a mixture of affective features from different diagnostic categories. Perhaps, in time, greater understanding of the psychiatric disorders specific to patients with neurological disorders will lead to establishing more distinct diagnoses for the affective disorders associated with TLE. Finally, it must be recognised that studies such as ours are not conducted in a vacuum, but take place in clinical settings in which treatment efforts rightly take precedence over research needs. Many of the participants in this investigation were receiving psychiatric treatment before and after anterior temporal lobectomy, making it difficult to completely capture the natural history of psychiatric function around the time of surgery.

The understanding and treatment of psychiatric disorders in patients with TLE undergoing resective surgery continues to pose a major challenge. It seems indisputable that enhanced psychiatric and psychosocial functioning depend on a very substantial reduction in seizures. However, it is equally apparent that abolishing seizures is not sufficient to ensure an improvement in the psychiatric status of these patients. Psychiatric problems in patients undergoing anterior temporal lobectomy are multifactorial and are determined by the pathology and location of the cerebral dysfunction underlying seizures, the medicines used to treat the epilepsy, genetic predisposition, social and family circumstances, as well as the surgery itself. Furthermore, the role of surgery in the psychiatric function of patients with TLE is not limited to its effects on seizures. Even when surgery successfully abolishes seizures and leads to improved long term psychosocial adaptation, during the few months immediately after surgery a significant number of 
patients are still at risk of developing new psychiatric symptoms. Multiple lines of investigation and intervention, therefore, are likely to be required to address the complexities of psychiatric dysfunction in patients with TLE.

Our thanks to Carrie E Plummer and Gerry Wilson for their help in data collection and analysis.

1 Blumer D, Montouris G, Hermann BP. Psychiatric morbidity in seizure patients on a neurodiagnostic monitoring unit. fournal of Neuropsychiatry 1995;7:445-56.

2 Fenwick PBC, Blumer D, Caplan R, et al. Presurgical psychiatric assessment, In: Engel J, ed. Surgical treatment of the epilepsies. New York, Raven Press, 1993:273-90.

the eplepsies. New York, Raven Press, 1993:273-90.
3 Jensen I, Larsen JK. Mental aspects of temporal lobe epilepsy: follow-up of 74 patients after resection of a epilepsy: follow-up of 74 patients after resection of a
temporal lobe. $\mathcal{F}$ Neurol Neurosurg Psychiatry 1979;42:25665 .

4 Koch-Weser M, Garron DC, Gilley DW, et al. Prevalence of psychologic disorders after surgical treatment of seizures. Arch Neurol 1988;45:1308-11:

5 Mendez M, Cummings JL, Benson DF. Depression in epilepsy: significance and phenomenology. Arch Neurol 1986;43:766-70.

6 Perini GI, Tosin C, Carraro C, et al. Interictal mood and personality disorders in temporal epilpesy and juvenile myoclonic epilepsy. I Neurol Neurosurg Psychiatry 1996;61: $601-5$.

7 Taylor DC. Mental state and temporal lobe epilepsy. Epilepsia $1972 ; 13: 727-65$

8 Victoroff J. DSM-III-R psychiatric diagnoses in candidates for epilepsy surgery: lifetime prevalence. Neuropsychiatry, Neuropsychology, and Behavioral Neurology 1994;7:87-97.

9 Walker AE, Blumer D. Behavioral effects of temporal lobectomy for temporal lobe epilepsy. In: Blumer D, ed. Psychiatric aspects of epilepsy. Washington, DC: American Psychiatric aspects of epilepsy. Washin
atric Press, 1984:295-323.

10 Perrine K, Hermann BP, Meador KJ, et al The relationship of neuropsychological functioning to quality of life in epilepsy. Arch Neurol 1995;52:997-1003.

11 Taylor DC. Psychiatric and social issues in measuring the input and outcome of epilepsy surgery. In: Engel J, ed. Sur gical treatment of the epilepsies. New York: Raven Press, 1987:485-503.

12 Victoroff J, Benson DF, Grafton ST, et al. Depression in complex partial seizures. Arch Neurol 1994;511:155-63.

13 Blumer D, Wakhlu S, Davies K, et al. Psychiatric outcome of temporal lobectomy for epilepsy: incidence and treatment of psychiatric complications. Epilepsia 1998;39:478-86.

14 Ring HA, Moriarty J, Trimble MR. A prospective study of the early postsurgical psychiatric associations of epilepsy
surgery. F Neurol Neurosurg Psychiatry 1998;64:601-4.

15 Hermann BP, Wyler A, Somes G. Preoperative psychological adjustment and surgical outcome are determinants of psychosocial status after anterior temporal lobectomy. $\mathcal{F}$ Neurol Neurosurg Psychiatry 1992;55:491-6.

16 Hermann BP, Wyler AR, Ackerman B, et al. Short-term psychological outcome of anterior temporal lobectomy. $\mathcal{F}$ psychological outcome of

17 Trenerry M, Hermann BP, Barr W, et al. MMPI scale elevations before and after right and left temporal lobectomy. Assessment 1996;3:307-15.
18 Altshuler LL, Devinsky O, Post RM, et al. Depression, anxiety, and temporal lobe epilepsy. Arch Neurol 1990;47:2848.

19 Bear DM, Fedio P. Quantitative analysis of inteictal behavior in temporal lobe epilepsy. Arch Neurol 1977;34:454-67.

20 Sperling MR, O'Connor MJ. Comparison of depth and subdural electrodes in recording temporal lobe seizures. Neurology 1989;39:1497-504.

21 Sperling MR, O'Connor MJ, Saykin AJ, et al. A non-invasive protocol for anterior temporal lobectomy. Neurology 1992; 42:416-22.

22 American Psychiatric Association. Diagnostic and statistical manual of mental disorders, revised 3rd edition. Washington, DC: American Psychiatric Association, 1987.

23 Spitzer RL, Williams JBW, Gibbon M, et al. The structured clinical interview for DSM-III-R (SCID): history, rationale, and description. Arch Gen Psychiatry 1992:49:624-9.

24 Williams JBW, Gibbon M, Spitzer RL, et al. The structured clinical interview for DSM-III-R (SCID). II: Multisite testretest reliability. Arch Gen Psychiatry 1992;49:630-6.

25 Overall JE, Gorham DR. The brief psychiatric rating scale. Psychol Rep 1962;10:799-812.

26 Spielberger C. State trait anxiety inventory. Palo Alto, CA: Consulting Psychologists, 1983.

27 Beck AT, Ward CH, Mendelson M, et al. An inventory for measuring depression. Arch Gen Psychiatry 1961;4:561-71.

28 McNair DM, Lorr M, Doppellman LF. Profile of mood states. San Diego: Educational and Industrial Testing Service, 1992.

29 Trimble MR. Behaviour change following temporal lobectomy, with special reference to psychosis. F Neurol Neurosurg Psychiatry 1992;55:89-91.

30 Zwil AS, McAllister TW, Cohen I, et al. Ultra-rapid cycling bipolar affective disorder following a closed-head injury. Brain Injury 1993;7:147-52.

31 Engel J. Outcome with respect to epileptic seizures. In: Engel J, ed.Surgical treatment of the epilepsies. New York: Raven Press, 1987:553-71.

32 Naylor AS, Rgvi-Hansen B, Kessing L, et al. Psychiatric morbidity after surgery for epilepsy: short term follow up of patients undergoing amygdalohippocampectomy. F Neurol Neurosurg Psychiatry 1994;57:1375-81.

33 Bladin PF. Psychosocial difficulties and outcome after temporal lobectomy. Epilepsia 1998;33:898-907.

34 Chovaz CJ, McLachlan RS, Derry PA, et al. Psychosocial function following temporal lobectomy: influence of seizure control and learned helplessness. Seizure 1994;3:171-6.

35 Rausch R, Crandall PH. Psychological status related to surgical control of temporal lobe seizures. Epilepsia 1982:23: 191-202.

36 Trimble MR. Behaviour changes following temporal lobectomy, with special reference to psychosis. I Neurol Neurosurg Psychiatry 1992;55:89-91

37 Flor-Henry P. Psychosis and temporal lobe epilepsy. Epilepsia 1969;10:363-95.

38 Flor-Henry P. Determinants of psychosis in epilepsy: a controlled investigation. Biol Psychiatry 1983;8:81-97.

39 Liotti M, Tucker DM. Emotion in asymmetric corticolimbic networks. In: Davidson RJ, Hugdahl K, eds. Brain asymmetry. Cambridge, MA: The MIT Press, 1995:389-423.

40 American Psychiatric Association. Diagnostic and statistical manual of mental disorders-DSM-IV. Washington, DC: APA, 1994 